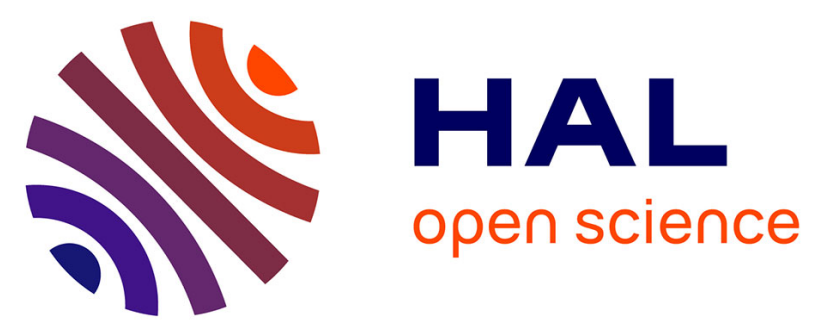

\title{
Instant Controlled Pressure Drop technology: From a new fundamental approach of instantaneous transitory thermodynamics to large industrial applications on high performance-high controlled quality unit operations
}

\author{
Sabah Mounir, Tamara Allaf, Baya Berka, Aicha Hassani, Karim Allaf
}

\section{To cite this version:}

Sabah Mounir, Tamara Allaf, Baya Berka, Aicha Hassani, Karim Allaf. Instant Controlled Pressure Drop technology: From a new fundamental approach of instantaneous transitory thermodynamics to large industrial applications on high performance-high controlled quality unit operations. Comptes Rendus. Chimie, 2014, 17, pp.261-267. 10.1016/j.crci.2013.10.019 . hal-02635254

\section{HAL Id: hal-02635254 \\ https://hal.inrae.fr/hal-02635254}

Submitted on 27 May 2020

HAL is a multi-disciplinary open access archive for the deposit and dissemination of scientific research documents, whether they are published or not. The documents may come from teaching and research institutions in France or abroad, or from public or private research centers.
L'archive ouverte pluridisciplinaire HAL, est destinée au dépôt et à la diffusion de documents scientifiques de niveau recherche, publiés ou non, émanant des établissements d'enseignement et de recherche français ou étrangers, des laboratoires publics ou privés. 


\title{
Instant Controlled Pressure Drop technology: From a new fundamental approach of instantaneous transitory thermodynamics to large industrial applications on high performance-high controlled quality unit operations
}

\author{
Sabah Mounir ${ }^{\text {a,b }}$, Tamara Allaf ${ }^{c, d}$, Baya Berka ${ }^{\text {a,e }}$, Aicha Hassani ${ }^{\text {e }}$, Karim Allaf ${ }^{\text {a,* }}$ \\ a University of La Rochelle - LASIE 3474 FRE - CNRS, 17042 La Rochelle cedex 01, France \\ ${ }^{\mathrm{b}}$ Zagazig University, Faculty of Agriculture, 44115 Zagazig, Egypt \\ ${ }^{c}$ ABCAR-DIC Process, BP 12053, 17010 La Rochelle cedex 01, France \\ d University of Avignon - INRA, UMR 408, 84000 Avignon, France \\ e École Normale Supérieure de Kouba, BP 92, Vieux-Kouba, 16500 Alger, Algeria
}

Keywords:

Solvent Extraction

Process Intensification

Instant Controlled Pressure Drop DIC

Starting accessibility $\delta X_{\mathrm{s}}$

Effective diffusivity $D_{\text {eff }}$

Yields

Availability ratio

\begin{abstract}
A B S T R A C T
Solvent extraction processes have been largely used in various industries. They recently were improved through new physical concepts such as $\mathrm{CO}_{2}$ Supercritical Fluid Extraction, Ultrasound assisted process, Microwave-assisted extraction, Instant Controlled Pressure Drop DIC-assisted extraction... Systematically, a pretreatment stage of grinding takes place in order to improve the exchange surface increasing the starting accessibility. Swelling of the material structure implies an increase of the porosity thus leading to higher solvent diffusivity within the solid matrix. A new concept of expanded granule powder has recently been defined using Instant Controlled Pressure Drop DIC technology. Whatever the type of solvent is (even $\mathrm{CO}_{2}$-SFE), such a swelled structure dramatically intensifies the kinetics through a higher specific exchange surface thanks to the open pores, while improving the solution solvent-solute diffusivity within the solid. Coupled to ultrasound, the internal transfer of solute within the pore solvent can likewise be intensified by replacing molecular diffusion within the pores by an effective convection transfer. In this work, we carried out a first approach of modeling of solvent extraction kinetics of expanded granules involving higher exchange surface and greater internal diffusion process.
\end{abstract}

\section{Introduction}

The main objective of different research works carried out on industrial unit operations is to improve both process performance and final product quality. It means to face the problematic of time and energy consumption, while preserving and even improving multi-criteria quality. The global intensification philosophy consists in

\footnotetext{
* Corresponding author.

E-mail address: kallaf@univ-lr.fr (K. Allaf).
}

listing the different processes, determining their possible interaction and considering between the main successive processes, the limiting phenomena. This last should be intensified to improve the global kinetics of the operation. Thus, while empirical laws allow controlling operating parameters depending on various process performance and final product quality parameters used as responses (dependent variables) [1], only fundamental studies can imply defining intensification processes.

Fundamental and experimental studies were carried out in the case of various conventional unit operations. When mass transfer achieved through internal diffusion is 
the limiting process, Instant Controlled Pressure Drop (DIC) can systematically contribute to defining new innovative intensified operations. The expanded structures as well as the instantaneous autovaporization induced by DIC are normally very effective. Other experiments and operations using such types of instantaneous transformations can systematically be more effective than quasistatic transformations. From a fundamental point of view, it was highlighted the inadequacy of the conventional quasi-static thermodynamic laws of mass transfer phenomena to explain and control a main part of the transfer phenomena achieved through instantaneous ways. Three thermodynamic laws of instant phenomena were proposed [2]:

- when a complex transformation is achieved instantaneously, it generates a null total entropy;

- various thermodynamic parameters have different priorities while reaching the equilibrium state;

- thus, a one-variable transformation cannot be achieved in an instantaneous way.

Thermodynamic analysis of various transformations should usually consider system particles with permanent, isotropic and random movement. The specific internal fluctuation kinetic energy reveals the system temperature. In some very limited cases of the instantaneous operations, such as DIC, the system particles can have a transitory anisotropic and unrandom/one-axis movement, during a very short time. It normally lasts for some milliseconds, where only the part of the 2-other axis of internal kinetic energy should concern the internal fluctuation; temperature is then observed to be much lower [3]. During this transitory stage, achieved by instantaneously dropping the pressure, the system temperature is much lower than the equilibrium level and the autovaporization ratio is much superior. Such phenomenon has been proved to be very effective in numerous industrial operations. Without increasing the energy consumption, the amount of evaporated volatile compounds is then much higher $[4,5]$.

Thus, by achieving instantaneously a pressure drop, the temperature decreases from its initial level $T_{i}$, to cross and be at $T_{f}$ lower than the equilibrium level determined by conventional quasi-static thermodynamics: $T_{f}<T_{s}$. Consequently, the lower the value of $T_{f}$, the higher the amount of vapor $m_{v}$ generated by autovaporization (Eq. 1). The evolution of the system from the lowest temperature $T_{f}$ towards the equilibrium level $T_{s}$ is accomplished respecting usual non-equilibrium quasi-static thermodynamics:

$m_{v}=\frac{m_{s}\left(c_{v_{s}}+W c_{v_{w}}\right)\left(T_{f}-T_{i}\right)}{L}$

In some specific cases, the cooling is so instant that it would preserve the molecular high temperature structure of the solid [2].

These different fundamental studies were approved through experimental approaches. The instantaneity laws, strictly linked to a specific bifurcation, contribute to carry out specific intensification processes. Various industrial unit operations were achieved coupling controlled high quality final products to the best performance of the

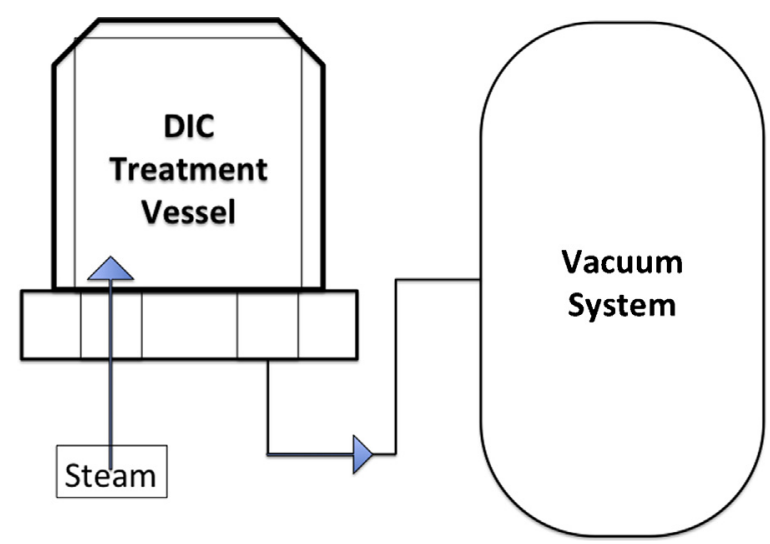

Fig. 1. Scheme of lab-scale DIC equipment.

operation (energy consumption, environment impact, great kinetics...) [6-8].

\section{DIC treatment in solvent extraction}

DIC treatment is based on the thermo-mechanical effects issued from an abrupt pressure drop $(\Delta P)$ $\Delta t>0.5 \mathrm{MPa} / \mathrm{s}$ ) towards a vacuum (about $5 \mathrm{kPa}$ ) taking place after a short-time/high-temperature and pressure stage (Figs. 1 and 2). In the processes of extraction of volatiles, DIC can be mainly used as an autovaporization procedure $[5,6,9,10]$.

The abrupt pressure drop causes instant and reinforced cooling of the products, immediately stopping thermal degradation, swelling the texture and even breaking cell walls, allowing valuable compounds to be more available and accessible. This operation completely modifies the technological abilities of various plants regarding heat and mass transfers. Powders issued from these technologies are distinguished by their specificity as expanded granule texture, which enables them to get very high functional behavior. Open pores increase the exchange surface and, thus, the starting accessibility. While the new porosity issued from the repartition of small pores implies higher effective diffusivity $[11,12]$.

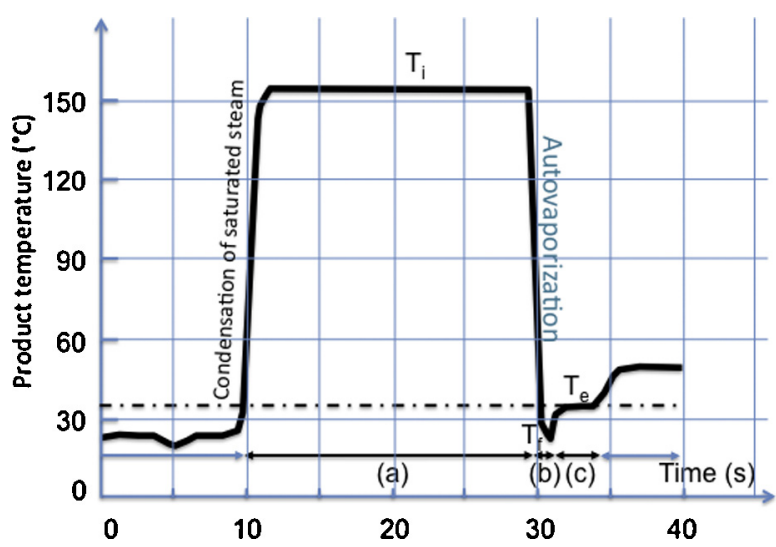

Fig. 2. Scheme of DIC process. 
Version définitive du manuscrit publiée dans / Final version of the manuscript published in :

Comptes Rendus Chimie (2014), Vol. 17, p. 261-267, DOI: 10.1.016/j.crci.2013.10.019

\section{Journal homepage: www.sciencedirect.com}

Indeed, the DIC expansion enables the vegetal materials to be much more adapted to solvent extraction; even supercritical $\mathrm{CO}_{2}$ becomes dramatically intensified $[1,13,14]$. The DIC-assisted extraction processes can be used as a system of both autovaporization and expansion aspects [14]. The first allows the volatile compounds (essential oils...) to be directly extracted in some minutes ( 1 to $4 \mathrm{~min}$ ) instead of some hours with conventional methods and some dozens of minutes with some "innovative" (microwave...) methods. Since the internal high total pressure is much higher than the external vacuum pressure, the main part of the evaporated molecules is transported towards the surrounding medium using Darcy's law with the gradient of the total pressure as driving force $[2,15]$. The final essential-oil-inwater stable emulsion we obtained by DIC can be a good basis for the conventional normal or vacuum distillation (liquid-vapor interaction), for some specific uses. This essential oil emulsion DIC can also be a good basis for encapsulation process.

Using DIC as a pretreatment-expanding process, one can greatly intensify the solid-fluid interactions through the definition of new phenomena such as the expulsion by forcing out, the expansion preparing the product to be more adequate to pressing and by increasing the diffusivity of water and solvents within the solid matrix [8]. DIC expansion enables the vegetal materials to be much more adapted to mass transfer.

In terms of drying, coupled to conventional airflow drying, optimized DIC treatment could completely remedy the shrinkage effect, increase the kinetics of the second stage of drying and improve the total quality of the final products. Airflow drying coupled to DIC treatment has allowed defining the new operation of swell-drying, used for producing high quality, low energy-consuming dried fruits and vegetables [12,16-18].

Another drying operation includes the properly said autovaporization; it can be used to define a very effective intermittent operation: Multi-Flash Drying MFD or Dehydration by Successive Pressure Drops DDS [19,20]. Both MFD and DDS operations are relevant answers to the paradoxical stage of various drying and Freeze-Drying operations. Once the product reaches the glass-transition $T_{\mathrm{g}}$, the dehydration mainly of the desorption stage can be achieved through DDS, which allows the vapor to remove using the gradient of total pressure as driving force (Darcy type mass transfer). Total drying time is then much lower as well as the energy consumption.

DIC treatment is an exceptionally relevant process for the microbiological decontamination of powders and solid materials. Its effects are both thermal (similar to UHT treatment) and mechanical (expansion and explosion of spores and vegetative microorganisms) [21]. The three operating parameters of temperature, treatment time and number of cycles can be used to define this decontamination level.

\section{Fundamental approach of solvent extraction}

Before studying any intensification operation, one has to study the fundamentals of extraction kinetics, trying to identify the limiting phenomenon, which firstly has to be improved.

\subsection{Mechanisms of mass transfers}

It is well known that the process of solvent extraction has four physical mechanisms of mass transfer:

- solvent interaction with the material exchange surface;

- solvent transfer within the product; it is carried out in liquid form by various processes, including capillarity, molecular diffusivity; the gradient of solvent content is the driving force;

- solute transfer in the solvent within the product pores; it is carried out by molecular diffusivity; the gradient of its concentration in the solvent is the driving force;

- solute transport from the product surface to the external solvent outside. When no external agitation is operated on the solvent, this transport takes place by diffusion with the gradient of solute concentration in the solvent as the driving force. By stirring, this transport is done by convection.

The appropriate solvent is normally identified based on polarity, toxicity...; it is usually able to instantaneously dissolve the solute. The choice of the solvent (and the adequate temperature) may allow assuming the interaction between solvent and product to put instantaneously the solute in solution.

The agitation of the external environment solvent allows the part of the solute $m_{\mathrm{A}}$ accessible at the exchange surface to be easily and quickly extracted and transported from the exchange surface to the outside by convection. The part of solute to be transferred by diffusion within the material and inside the solvent is considered to be $\left(m_{\mathrm{i}}-m_{\mathrm{A}}\right)$. A residual part $m_{\mathrm{r}}$ of this deep solute may be unavailable for extraction because of its location within cells with great wall and membrane resistance.

In terms of solute densities ( $\mathrm{g}$ of solute/100 g Dry Basis or \% $\mathrm{db}$ ), one can consider:

- $X_{s}$ : corresponds to $m_{s}$. It is the part of the solute located at the surface and initially accessible, quickly removed, mainly by convection thanks to the solvent agitation by interaction between the solvent and the surface of the product;

- $\left(X_{i}-X_{s}\right)$ : corresponds to the initial amount of the solute $\left(m_{i}-m_{s}\right)$ firstly located as a supposed uniform and homogeneous density within the volume. It evolves over time thanks to various diffusion processes. At time $t$, it is indicated by $X$;

- $\left(X_{i}-X_{\infty}\right)$ : corresponds to the amount of residual solute $m_{\mathrm{r}}$ unavailable for the extraction ( $\mathrm{kg}$ of solute per $\mathrm{kg}$ of dry matter or \% db).

\subsubsection{Washing stage of extraction process}

Once the material granule is solvent logged, the extraction process begins by a starting fast extraction step [22]. Allaf [15] defined this starting step as the washing stage of the surface-solvent interaction (Fig. 3): 
Version définitive du manuscrit publiée dans / Final version of the manuscript published in :

Comptes Rendus Chimie (2014), Vol. 17, p. 261-267, DOI: 10.1016/j.crci.2013.10.019

Journal homepage: www.sciencedirect.com

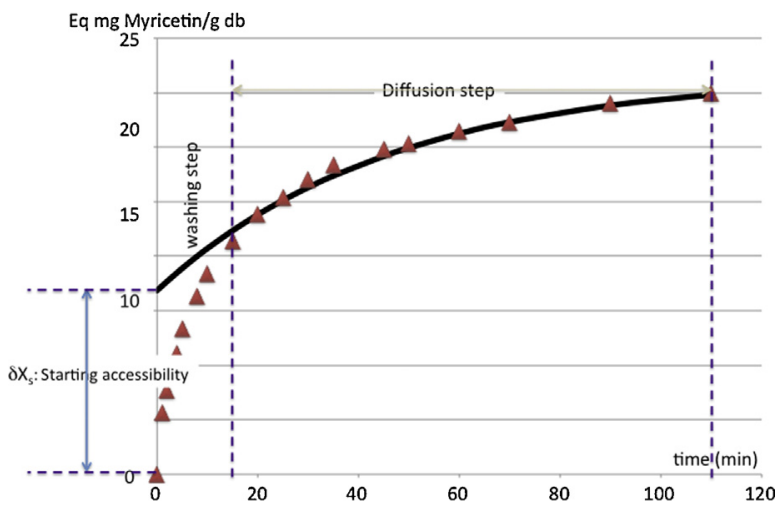

product must present the highest transfer resistance. In such conditions, the mass transfer within the expanded granule controls the extraction kinetics; it is given by Allaf's presentation of similar Fick diffusion [2]:

General Fick mass transfer law:

$\frac{\rho_{s}}{\rho_{m}}\left(\vec{v}_{s}-v_{m}\right)=-D_{e f f} \cdot \vec{\nabla}\left(\frac{\rho_{s}}{\rho_{m}}\right)$

with:

$\rho_{\mathrm{s}}$ : apparent density of solute in the material $\left(\mathrm{kg} \mathrm{m}^{-3}\right)$,

$\rho_{\mathrm{m}}$ : apparent density of dry material $\left(\mathrm{kg} \mathrm{m}^{-3}\right)$,

$\overrightarrow{v_{s}}$ : absolute velocity of solute flow within the porous medium $\left(\mathrm{m} \mathrm{s}^{-1}\right)$.

$\overrightarrow{v_{m}}$ : absolute velocity of solid medium (in $\mathrm{m} \mathrm{s}^{-1}$ ).

$D_{\text {eff: }}$ : effective diffusivity of solvent within the solid

Fig. 3. Kinetics of extraction of flavonoids from buckthorn leaves: phenomenological modeling including washing step and diffusion step. Model parameters are $D_{\text {eff }}$ : the effective diffusivity $\left(\mathrm{m}^{2} \mathrm{~s}^{-1}\right) ; \delta X_{\mathrm{s}}$ and $X_{\infty}$ : the starting accessibility and the yields or availability ratio, respectively (Eq mg Myricetin/g db).

Extraction rate of solute from surface:

$\frac{\mathrm{d} m_{s}}{\mathrm{~d} t}=k_{e} E S A\left(\varpi_{s}-\varpi_{\text {solvent }}\right)$

where:

$m_{s}$ : mass of solute extracted by interaction between the solvent and the surface of the product ( $\mathrm{kg}$ of solute);

$k_{e}$ : interaction coefficient between the solvent and the surface, by diffusion for the static case or by convection usually due to stirring ( $\mathrm{kg}$ of solvent $\mathrm{m}^{-2} \mathrm{~s}^{-1}$ );

ESA: exchange surface area between solvent and the product $\left(\mathrm{m}^{2}\right)$;

$\varpi_{s}$ : dissolving coefficient at equilibrium ( $\mathrm{kg}$ of solute $\mathrm{kg}^{-1}$ of solvent);

$\varpi_{\text {solvent }}$ : solute concentration in the surrounding solvent in contact with the external material surface ( $\mathrm{kg}$ of solute $\mathrm{kg}^{-1}$ of solvent).

The density of the extraction rate of solute from the surface per unit of dry matter:

$\frac{\mathrm{d} X_{s}}{\mathrm{~d} t}=k_{e} \operatorname{SESA}\left(\varpi_{s}-\varpi_{\text {solvent }}\right)$

SESA: specific exchange surface area between the solvent and the product per unit of dry basis $\left(\mathrm{m}^{2} \mathrm{~kg}^{-1}\right)$.

The total effect can be shown through the "starting accessibility" parameter, preferentially using an effective specific exchange surface area SESA eff: :

Starting accessibility:

$\delta X_{s}=\mathrm{k}_{e} \operatorname{SESA}_{\text {eff }}\left(\varpi_{e}-\varpi_{\text {solvent }}\right) \delta t$

Usually, in kinetics studies, the starting accessibility $\delta X_{s}$ does not involve any diffusion source or effect; it is the density of solute per unit of dry matter extracted by the initial interaction between the solvent and the effective exchange surface of the product. $\delta X_{s}$ is expressed as $\mathrm{kg}$ of solute per $\mathrm{kg}$ of dry matter or $\% \mathrm{db}$.

\subsubsection{Internal transfer by diffusion}

After the first and quick washing stage of extraction of superficial solute, the internal mass transfer within the medium (in $\mathrm{m}^{2} \mathrm{~s}^{-1}$ ).

By neglecting the possible shrinkage or swelling process, one can assume that $\rho_{m}=$ constant and $\overrightarrow{v_{m}}=0$, it is possible to have:

Simplified Fick mass transfer law:

$\rho_{s} \overrightarrow{v_{s}}=-D_{e f f} \cdot \vec{\nabla} \rho_{s}$

Using the balance mass, it is possible to obtain:

Second Fick mass transfer law:

$\frac{\partial \rho_{s}}{\partial \mathrm{t}}=\vec{\nabla}\left(D_{e f f} \vec{\nabla} \rho_{s}\right)$

where $t$ is the time.

Although the effective diffusivity $D_{\text {eff }}$ considerably varies versus the system temperature and porosity, it can be assumed to be constant only through the hypothesis of both structural and thermal homogeneities. By estimating physical processes and carrying out fitting experiments one can normally confirm this hypothesis allowing the equation to become the second Fick's law:

$\frac{\partial \rho_{s}}{\partial t}=D_{e f f} \nabla^{2} \rho_{s}$

And, for a unidirectional radial flow, it becomes:

$\frac{\partial \rho_{s}}{\partial t}=D_{e f f} \frac{\mathrm{d}^{2} \rho_{s}}{\mathrm{~d} r^{2}}$

The solutions to provide to this diffusion equation closely depend on the initial and boundary conditions. The Crank's solution [23] according to the geometry of the spherical expanded granule may be adopted, and by using the amount of extract, it is possible to get:

$\frac{X_{\infty}-X}{X_{\infty}-X_{A}}=\sum_{1}^{\infty} A_{i} \mathrm{e}^{-q_{i}^{2}\left(t-t_{0}\right)}$

where $X$ is the amount of solute extracted at time $t$, and $X_{\infty}$ is the amount of solute extracted at time $t \rightarrow \infty$ (total yields). $X_{\mathrm{A}}$ is an amount of solute extract at time $t=t_{\mathrm{o}}$, which is chosen as a time for which the starting washing step has completely disappeared and the diffusion process is assumed to be the highest resistance of the solvent extraction. By using the experimental data for the diffusion model, one has to exclude the value near the starting point $\left(t<t_{\mathrm{o}}\right)$. $\delta X_{\mathrm{s}}$ is then calculated by extrapolating $($ at $t=0)$ this 
diffusion model. The value $\delta X_{\mathrm{s}}$ would correspond to the amount of solute accessible on the surface and extracted from it in a very short time. By modifying granule structure, improving porosity, breaking the cell wall... the values of $X_{\infty}$ (yields) and $\delta X_{s}$ (starting accessibility), as well as the effective diffusivity $D_{\text {eff }}$ vary to be considered as the main response parameters characterizing the intensification treatment.

$$
\begin{aligned}
\frac{X_{\infty}-X}{X_{\infty}-X_{A}}= & \frac{6}{\pi^{2}} \mathrm{e}^{-\frac{\pi^{2} D_{\text {eff }}}{r^{2}}\left(t-t_{0}\right)}+\frac{6}{4 \pi^{2}} \mathrm{e}^{-\frac{4 \pi^{2} D_{\text {eff }}}{r^{2}}\left(t-t_{0}\right)} \\
& +\frac{6}{9 \pi^{2}} \mathrm{e}^{-\frac{9 \pi^{2} D_{\text {eff }}}{r^{2}}\left(t-t_{0}\right)}+\ldots
\end{aligned}
$$

Which can be limited to its first term, to be expressed as:

$$
\frac{X_{\infty}-X}{X_{\infty}-X_{A}}=A \mathrm{e}^{-k\left(t-t_{0}\right)}
$$

By using the logarithmic representation, this equation may be transformed into a straight line with a slope $k$ from which $D_{\text {eff }}$ could be determined:

$D_{\text {eff }}=\frac{r^{2} k}{\pi^{2}}$

The experimental data used for such a diffusion model exclude the ones included within the washing step (experimental points close to $t=0$ ). Thus, the extrapolation of the diffusion model till $t=0$ allows determining a value $X_{\mathrm{o}}$, generally different from the initial experiment value $X_{(t=0)}=0$. Thus, $\delta X_{S}=\left(X_{0}-X_{(t=0)}\right)=X_{0}$ gives the solutes quickly removed from the surface (Fig. 3 ), independently from diffusion processes.

\subsection{Intensification processes}

Various technologies such as grinding, DIC expansion, ultrasounds. . . can be envisaged as intensifying processes, depending on the steps and phenomenon to be improved.

DIC, used as a pretreatment-expanding process, can greatly intensify the extraction kinetics and yields through the definition of new phenomena such as the expulsion by forcing out, the expansion preparing the product to be more adequate to grinding and by increasing the diffusivity of solvents within the solid matrix.

\subsubsection{Intensification of the washing stage}

Extraction processes can be enhanced by grinding the raw material: the smaller the average granule size, the higher the specific surface exchange area (SESA).

A sufficiently strong solvent stirring-agitation can also intensify the solvent-surface interaction thanks to a convection exchange instead of a molecular diffusion mass transfer; the dissolution coefficient of the solute by the solvent $\left(k_{e}\right)$ is increased, and the value of $\varpi_{\text {solvent }}$ becomes lower. This amplifies the kinetics of the process compared to a simple static-solvent process. An appropriate ultrasound treatment can also help increase the internal fluctuation motion of the particles and the solute flow from the surface towards the external medium.
Another effective intensification method can be obtained by injecting solvent after an initial vacuum step. The open and dead-end pores are normally filled with air, which acts as an insulating medium. However, an initial vacuum stage prior to the injection of solvent would modify the initial situation and almost completely fill these pores. Thanks to the initial vacuum step there is a close contact and a more effective exchange between the "total surface" and the solvent, thus avoiding the limitation of the geometrically smooth surface. The effective exchange surface area between the solvent and the product is now much higher. After the vacuum step, K. Allaf and T. Allaf [1] identified the density of the extraction rate of solute from the surface per unit of dry matter (Eqs. 3 and 4) to become much higher.

"External" intensification processes are generally easy to carry out. Once the external transfer phenomenon is perfectly intensified, it is no longer the limiting process. Solute extraction kinetics from the surface can then reach its highest level.

Since the "starting accessibility" $\delta X_{\mathrm{s}}$ directly depends on the effective specific surface exchange area SESA $A_{\text {eff- }}$ Thus the lower the granulometry, the higher the starting accessibility.

\subsubsection{Intensification of the diffusion stage of solvent extraction}

The external modification such as the use of strong agitation after grinding and/or the establishing an initial vacuum state only modifies the starting accessibility $\delta X_{\mathrm{s}}$. It doesn't affect the effective diffusivity value, but it does reduce the time frame needed for the internal solute extraction. Thus the intensification of the diffusion stage of solvent extraction operation implies:

- a grinding process, which normally allows the diffusion size in the material to reduce;

- a stirring allowing solute transport from the product surface to outside to be carried out by convection;

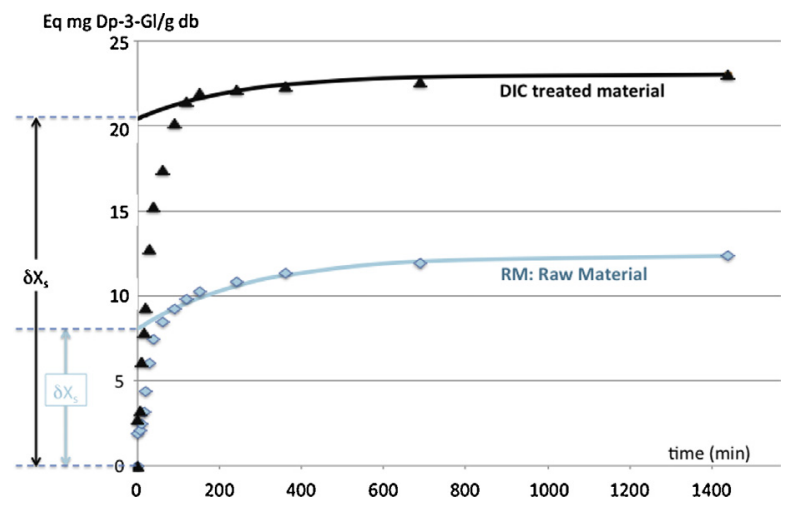

Fig. 4. Intensification of total anthocyanin extraction from Buckthorn leaves (Raw material and DIC treated material): phenomenological modeling of extraction kinetics including washing step and diffusion step. Kinetic modeling parameters: $\delta X_{s}$ : the starting accessibility (Eq mg Dp-3$\mathrm{Gl} / \mathrm{g} \mathrm{db}), D_{\text {eff: }}$ The effective diffusivity $\left(\mathrm{m}^{2} \mathrm{~s}^{-1}\right)$, and $X_{\infty}$ : the yields or availability ratio (Eq $\mathrm{mg} \mathrm{Dp}-3-\mathrm{Gl} / \mathrm{g} \mathrm{db}$ ). 
Version définitive du manuscrit publiée dans / Final version of the manuscript published in :

Comptes Rendus Chimie (2014), Vol. 17, p. 261-267, DOI: 10.1016/j.crci.2013.10.019

Journal homepage: www.sciencedirect.com

Table 1

Impacts of buckthorn leaf texture (raw material and DIC-treated material) as intensification process of total anthocyane extraction, in terms of starting accessibility $\delta X_{\mathrm{s}}$ (mg Gallic Acid Equivalent GAE/g db), effective diffusivity $D_{e f f}\left(\mathrm{~m}^{2} \mathrm{~s}^{-1}\right)$, and the yields or availability ratio $X_{\infty}(\mathrm{mg}$ GAE/g db) versus the saturated steam pressure $P(\mathrm{MPa})$, the total thermal treatment time (s), and the number of cycles $N$.

\begin{tabular}{llll}
\hline DIC conditions & $\begin{array}{l}P=0.5 \mathrm{MPa} ; t=180 \mathrm{~s} ; \\
N=5 \text { cycles }\end{array}$ & $\begin{array}{l}P=0.1 \mathrm{MPa} ; t=120 \mathrm{~s} ; \\
N=4 \text { cycles }\end{array}$ & Raw material \\
\hline $\begin{array}{l}\text { Starting accessibility } \\
\delta X_{\mathrm{s}}(\mathrm{mg} \text { GAE} / \mathrm{g} \mathrm{db})\end{array}$ & 1.18 & 2.04 & 0.81 \\
$\begin{array}{l}\text { Starting accessibility } \\
(\%) \text { versus raw material }\end{array}$ & $147 \%$ & $254 \%$ & $100 \%$ \\
$\begin{array}{l}\text { Diffusivity } \\
\left(10^{-12} \mathrm{~m}^{2} / \mathrm{s}\right)\end{array}$ & 2.54 & 1.72 & 0.99 \\
$\begin{array}{l}\text { Diffusivity improvement } \\
(\%) \text { versus raw material }\end{array}$ & $257 \%$ & $174 \%$ & $100 \%$ \\
$\begin{array}{l}\text { Availability or yields } X_{\infty} \\
\text { (mg GAE/g db) }\end{array}$ & 1.44 & 2.30 & 1.24 \\
$\begin{array}{l}\text { Availability or yields } X_{\infty} \\
(\%) \text { versus raw material }\end{array}$ & $116 \%$ & $186 \%$ & $100 \%$ \\
\hline
\end{tabular}

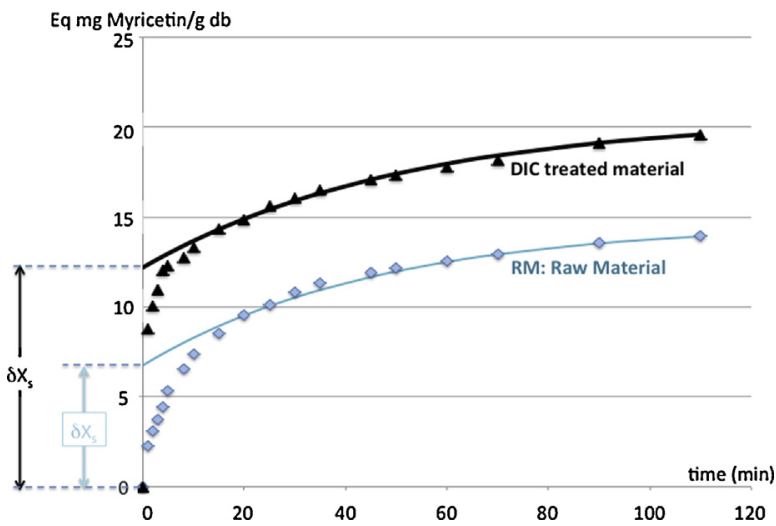

Fig. 5. Intensification of total flavonoid extraction from myrtle leaves (raw material RM and DIC treated material): phenomenological modeling of extraction kinetics with washing and diffusion steps. Kinetic modeling parameters: $D_{\text {eff: }}$ : the effective diffusivity $\left(\mathrm{m}^{2} \mathrm{~s}^{-1}\right), \delta X_{\mathrm{s}}$ and $X_{\infty}$ : the starting accessibility and the yields or availability ratio, respectively (Eq mg Myricetin/g db).

- a new texturing in order to expand the granules. Indeed, natural structure of vegetables and more specifically the cytoplasmic membrane and the cellular wall should not support the liquid transfer processes. The structure resistance often seems to be the principal restrictive factor of the operation kinetics;

- an increase of porosity to improve the solute transfer diffusivity of the solvent within the product;

- a ultrasound treatment allowing the mass transfer of solute within the solvent present in the pore to be achieved following convection process instead of molecular diffusion.

The DIC treatment is usually designed as a very relevant intensification process in order to increase both the starting accessibility and the diffusivity (Figs. 4 and 5, Table 1). It also allows the grinding to be easier with expanded granule powder.

\section{Conclusion}

The present study has established how important the grinding into swelled-granule powders is for intensifying the solvent extraction kinetics. A specific model of solvent extraction was defined; it has been appropriate with the expansion of granule generally achieved by the instant controlled pressure drop DIC treatment. The external conditions can normally allow the solvent convection to assure a starting quick extraction from the surface. Just after, it was assumed the extraction to be controlled by the only internal diffusion mass transfer.

This modeling leads to identify the impacts of DIC texturing on extraction kinetics through the yields $X_{\infty}$, the amount of extract accessible at starting time $X_{\mathrm{o}}=\delta X_{\mathrm{s}}$ (normally by solvent convection at the surface), and $D_{\text {eff }}$ (the effective diffusivity). The values of $X_{\infty}, \delta X_{s}$, and $D_{\text {eff }}$ will have to be considered as the main response parameters characterizing the intensification through DIC treatments in terms of technological abilities of the material regarding solvent extraction technology.

\section{References}

[1] K. Allaf, T. Allaf, Instant Controlled Pressure Drop (D.I.C.) in Food Processing: From Fundamental to Industrial Applications, Springer, New York, 2013.

[2] K. Allaf, Transfer phenomena and industrial applications, Lebanese University, Faculty of Science, Beirut, 1982.

[3] I. Sulaiman, Process Engineering, La Rochelle University, La Rochelle, France, 2013.

[4] M. Kristiawan, V. Sobolik, K. Allaf, J. Chromatogr. A 1192 (2008) 306-318.

[5] B. Berka-Zougali, A. Hassani, C. Besombes, K. Allaf, J. Chromatogr. A 1217 (2010) 6134-6142.

[6] C. Besombes, N. Albitar, K. Allaf, O. Barkat, 38th International Symposium on Essential Oils - ISEO Graz (Autriche), 2007, p. 44.

[7] K. Allaf, in: F. Chemat (Ed.), Essential Oils and Aromas: Green Extraction and Application, New Delhi, 2009, pp. 85-121.

[8] K. Allaf, C. Besombes, B. Berka-Zougali, M. Kristiawan, V. Sobolik, T. Allaf, in: N. Lebovka, E. Vorobiev, F. Chemat (Eds.), Enhancing Extraction Processes in the Food Industry, CRC Press Taylor \& Francis Group, Dublin, Ireland, 2011, pp. 255-302.

[9] T. Allaf, V. Tomao, K. Ruiz, K. Bachari, M. ElMaataoui, F. Chemat, LWT Food Sci. Technol. 51 (2013) 111-119.

[10] M. Kristiawan, V. Sobolik, M. Al-Haddad, K. Allaf, Chem. Eng. Process. 47 (2008) 66-75

[11] C. Téllez-Pérez, M.M. Sabah, J.G. Montejano-Gaitán, V. Sobolik, C.A. Martínez, K. Allaf, Procedia. Eng. 42 (2012) 978-1003.

[12] S. Mounir, T. Allaf, A.S. Mujumdar, K. Allaf, Drying Technol. 30 (2012) 1508-1531.

[13] B. Ben Amor, K. Allaf, Food Chem. 115 (2009) 820-825.

[14] T. Allaf, V. Tomao, K. Ruiz, F. Chemat, Ultrason. Sonochem. 20 (2013) 239-246. 
Version définitive du manuscrit publiée dans / Final version of the manuscript published in :

Comptes Rendus Chimie (2014), Vol. 17, p. 261-267, DOI: 10.1.016/j.crci.2013.10.019

Journal homepage: www.sciencedirect.com

[15] T. Allaf, [PhD thesis], University of Avignon, UMR 408 - INRA, France, 2013.

[16] C. Téllez-Pérez, M.M. Sabah, J.G. Montejano-Gaitán, V. Sobolik, C.A. Martínez, K. Allaf, Procedia Eng. 42 (2012) 978-1003.

[17] N. Albitar, S. Mounir, C. Besombes, K. Allaf, Drying Technol. 29 (2011) 993-1001

[18] P. Setyopratomo, A. Fatmawati, P.D. Sutrisna, E. Savitri, K. Allaf, AsiaPacific J. Chem. Eng. (2011)

[19] M.F. Zotarelli, B.D.A. Porciuncula, J.B. Laurindo, J. Food Eng. 108 (2012) 523-531.

[20] J. Haddad, F. Juhel, N. Louka, K. Allaf, Drying Technol. 22 (2004) 457478.

[21] T. Allaf, C. Besombes, I. Mih, L. Lefevre, K. Allaf, Decontamination of Solid and Powder Foodstuffs using DIC Technology, in: M. Schmidt (Ed.), Advances in Computer Science and Engineering, InTech, Croatia, 2011.

[22] L. Rakotondramasy-Rabesiaka, J.L. Havet, C. Porte, H. Fauduet, Ind. Crops Prod. 29 (2009) 516-523.

[23] J. Crank, The mathematics of diffusion, 2nd edition, Clarendon Press, Oxford, 1975. 